\title{
British community pharmacists' views of physician-assisted suicide (PAS)
}

Timothy R G Hanlon, Marjorie C Weiss and Judith Rees University of Bristol and University of Manchester

\begin{abstract}
Objectives-To explore British community pharmacists'views on PAS, including professional responsibility, personal beliefs, changes in law and ethical guidance.

Design-Postal questionnaire

Setting-Great Britain

Subjects- $A$ random sample of 320 registered

full-time community pharmacists

Results-The survey yielded a response rate of 56\%. The results showed that $70 \%$ of pharmacists agreed that it was a patient's right to choose to die, with $57 \%$ and $45 \%$ agreeing that it was the patient's right to involve his/her doctor in the process and to use prescription medicines, respectively. Forty-nine per cent said that they would knowingly dispense a prescription for use in PAS were it to be legalised and $54 \%$ believed it correct to refuse to dispense such a prescription. Although 53\% believed it to be their right to know when they were being involved in $P A S, 28 \%$ did not. Most pharmacists (90\%) said that they would wish to see the inclusion of a practice protocol for PAS in the code of ethics of the Royal Pharmaceutical Society of Great Britain (CE-RPSGB) in the event of a change in the law on PAS. In addition, 89\% would wish to see PAS included in the Conscience Clause of the $C E-R P S G B$. Males were found to be significantly less likely to favour PAS than females $(p<0.05)$, as were those declaring an ethnic/religious background of consideration when dealing with ethical issues in practice compared with their counterparts $(p<0.00005)$.

Conclusion-Pharmacists view their professional responsibility in PAS to be more obligatory than a physician's, in having to provide the means for PAS. It is worrying that a proportion of the respondents prefer to remain in ignorance of the true purpose of a prescription for PAS; a finding at odds with current developments within the pharmaceutical profession. A practice protocol for PAS and an extension of the conscience clause should be considered in the event of PAS becoming legal. Such measures would allow the efficient provision of the pharmaceutical service whilst at the same respecting the personal beliefs of those who object to cooperating in the ending of a life.

(Fournal of Medical Ethics 2000;26:363-369)
\end{abstract}

Keywords: Professional ethics; pharmacy ethics; community pharmacy; bioethics; physician-assisted suicide; euthanasia

\section{Introduction}

On 10th December 1997 the Houses of Parliament could have become one of the few legislative bodies in the world to have legalised physician-assisted suicide (PAS), third only to the state of Oregon in America and the Northern Territories in Australia. ${ }^{1}$ The practice of PAS in the Netherlands, whilst tolerated, remains officially a criminal offence. ${ }^{2} \mathrm{MP}$ Joe Ashton's private member's bill, Doctor assisted dying, received little publicity in Great Britain, and yet the legalisation of PAS would almost certainly have had a profound effect on the medicolegal world and on British society.

In what has become a much debated ethical issue, the discussion about the morality of PAS has tended to focus on the patient who seeks PAS and the doctor who prescribes the means in the form of prescription medicines. Little attention has been given to the involvement of the pharmacist who dispenses the prescription medicines used to assist the patient in his/her suicide. Two notable studies have been conducted exploring pharmacists' views on PAS, both of which were American. Rupp and Isenhower undertook a national survey of 1,050 US pharmacists. ${ }^{3}$ Nearly three-quarters of respondents agreed that patients were sometimes justified in wanting to end their own lives. Almost half of the respondents approved of physicians' active participation in this process. Age and religious affiliation were found to be influential in the pharmacists' attitudes to PAS. The second study, by Vivian et al, was conducted amongst Michigan pharmacists. ${ }^{4}$ They found that $70 \%$ of respondents favoured the legal right of patients to commit suicide, although only $50 \%$ agreed that patients had the "moral or ethical right" to commit suicide. Like Rupp and Isenhower, Vivian found that age and religious affiliation were influential in the pharmacists' attitudes towards PAS.

These studies, while offering useful insights, are of less relevance to the UK because of the difference in legal and ethical systems. Currently, the code of ethics of the Royal Pharmaceutical Society of Great Britain (CE-RPSGB) contains a point of guidance entitled limitations of conscience, which is referred to as the conscience clause. ${ }^{5}$ This allows those pharmacists who object to dispensing certain medicines legitimately to refuse to do so. This clause currently covers drugs used to control fertility and conception or to terminate pregnancy.

However, recent years have seen the role of the pharmacist expand beyond mere drug supply. New 
roles include offering advice on minor ailments, monitoring repeat prescriptions and providing services to general practitioner (GP) practices regarding appropriate and cost-effective drug use. ${ }^{6}$ Pharmacists are involved as medicine managers concerned with optimising individual outcomes from pharmaceutical interventions. This ethos for managing drug therapy stems from the work of Hepler and Strand who define pharmaceutical care as the responsible provision of drug therapy for the purpose of achieving definite outcomes that improve the patient's quality of life. ${ }^{8}$ No longer is it acceptable for pharmacists to claim that they only dispense drugs. With pharmacists increasingly wishing to receive recognition for their role as drug experts, they must accept a commensurate level of legal responsibility for their actions. ${ }^{9}$ In the UK, a pharmacist was held liable for $45 \%$ of the damages awarded after a patient suffered gangrene from an overdose of Migril prescribed for migraine in overdose by the physician. ${ }^{10}$ In this case the judge ruled that the pharmacist owed a duty to the patient to ensure that drugs were correctly prescribed. Further, the pharmacist should have spotted the doctor's error and queried the prescription with the prescriber. ${ }^{10}$ While pharmacists have always had a professional responsibility to assure the safe and effective use of the medicine they supply, developments in the pharmacist's role have heightened the awareness of pharmacists' professional, moral and ethical responsibilities towards patients. How these complex factors interact for everyday pharmacist practitioners is the focus of this work. This study aimed to explore British community pharmacists' views on PAS on matters such as professional responsibility, personal beliefs, changes in law and ethical guidance.

\section{Method}

The questionnaire was developed from interviews with eight pharmacists from a diverse range of experiences in community pharmacy; it was divided into three sections. The first section contained a scenario or vignette outlining a case of PAS. The vignette was designed to illustrate a case of PAS that respondents could associate with their everyday practice as community pharmacists. The second section consisted of twenty attitudinal statements relating to PAS. These statements were subdivided into two groups. In the first group of sixteen statements, respondents were asked to assume that PAS was still illegal in the UK and to rate their level of agreement with the statement, using a Likert scale. The remaining four statements asked respondents to assume that the law had changed to allow PAS and were answered with "yes", "don't know" or "no". The third section asked for the demographic details of the respondent: sex, year of registration as a pharmacist and current practice setting. The respondents were also asked to indicate "yes" or "no" as to whether they had any formal involvement in ethics as a subject and whether their ethnic/religious background was a consideration in terms of how they dealt with ethical issues in everyday practice. Each respondent was then asked to make any additional comments. A copy of the questionnaire forms Appendix I at the end of this paper.

The questionnaire was piloted among a group of ten colleagues in the School of Pharmacy and Pharmaceutical Sciences at the University of Manchester. Most of these respondents were also practising pharmacists. They were asked to complete the questionnaire and critically assess it. This led to a few minor adjustments.

The survey was carried out from May to July 1998. The questionnaire was sent to a random sample of 320 full-time community pharmacists listed in the Register of Pharmaceutical Chemists held by the Royal Pharmaceutical Society of Great Britain (RPSGB). Only pharmacists listed as practising full-time were included in this study as it was felt that this group would be most interested in the subject and most up to date with issues of practice. Furthermore, pharmacists practising in hospital and other branches of pharmacy were excluded from this study as respondents from these branches of the profession were anticipated to be too small in number to allow a meaningful assessment of their views to be made. After four weeks nonrespondents were sent a reminder letter which included a copy of the questionnaire. After a further three weeks the remaining non-respondents were sent a final letter of reminder and a further copy of the questionnaire. Data were analysed using the SPSS (Statistics Package for Social Scientists) using Kruskal-Wallis one-way ANOVA, MannWhitney U-Wilcoxon Rank Sum test and Spearman's Rank correlation coefficient.

\section{Results}

THE RESPONDENTS

A total of 183 questionnaires were returned out of a total of 320 . Four were returned blank. This yielded a final usable response rate of $56 \%$. There were 115 male respondents $(64 \%)$ from an original sample of 203 males (63\%). Pharmacists who participated in the main survey were asked to provide their year of registration as a pharmacist in Great Britain. To compare these to non-responders the year of registration of each of the 141 nonrespondents was identified using the RPSGB Annual Register of Pharmaceutical Chemists (1998). The mean year of registration for both the respondents and non-respondents was 1983 $(\mathrm{SD}+/-10.87$ for respondents and $+/-10.25$ for non-respondents.) Assuming that many pharmacists register at the age of 22 , this would give a mean age of 37 years old.

Respondents were asked to indicate their current practice setting: independent (including owners and employees), a small multiple (30 shops or less) or a large multiple (30 or more shops). Approximately half $(48 \%)$ indicated that their current practice setting was an independent pharmacy. Most respondents (93.3\%) indicated that they had no interest or involvement in ethics. Four respondents left this section blank, whilst eight $(4.5 \%)$ 


\begin{tabular}{|c|c|c|c|c|c|}
\hline \multicolumn{2}{|c|}{ Statements } & \multirow{2}{*}{$\begin{array}{l}\begin{array}{l}\text { Strongly Agree } \\
\text { / Agree }\end{array} \\
125(70 \%)\end{array}$} & \multirow{2}{*}{$\begin{array}{l}\text { Unsure } \\
16(9 \%)\end{array}$} & \multirow{2}{*}{$\begin{array}{l}\begin{array}{l}\text { Strongly } \\
\text { Disagree / } \\
\text { Disagree }\end{array} \\
38(21 \%)\end{array}$} & \multirow{2}{*}{$\begin{array}{l}\begin{array}{l}\text { Total } \\
\text { number }\end{array} \\
179\end{array}$} \\
\hline 1. & A patient has the right to choose to end his/her own life & & & & \\
\hline 2. & $\begin{array}{l}\text { A patient has the right to choose to die with the assistance of } \\
\text { his/her physician }\end{array}$ & $102(57 \%)$ & $27(15 \%)$ & $58(28 \%)$ & 179 \\
\hline 3. & $\begin{array}{l}\text { It is appropriate for a patient to use prescription medicines to } \\
\text { commit suicide }\end{array}$ & $80(45 \%)$ & $39(22 \%)$ & $59(33 \%)$ & 178 \\
\hline 4. & $\begin{array}{l}\text { A prescription medicine should not be used to end a life } \\
\text { prematurely }\end{array}$ & $53(30 \%)$ & $36(21 \%)$ & $86(49 \%)$ & 175 \\
\hline 5. & $\begin{array}{l}\text { A physician has a professional responsibility to alleviate suffering } \\
\text { by issuing a prescription for PAS }\end{array}$ & $52(29 \%)$ & $45(25 \%)$ & $82(46 \%)$ & 179 \\
\hline 6. & $\begin{array}{l}\text { It is a physician's professional responsibility not to act in such a } \\
\text { way that a life is ended prematurely as in PAS }\end{array}$ & $61(34 \%)$ & $53(30 \%)$ & $65(36 \%)$ & 179 \\
\hline 7. & $\begin{array}{l}\text { A pharmacist has a professional responsibility to dispense a } \\
\text { prescription to be used in PAS, if that is what the patient wants }\end{array}$ & $82(46 \%)$ & $30(17 \%)$ & $66(37 \%)$ & 178 \\
\hline 8. & $\begin{array}{l}\text { It is inappropriate for a pharmacist to provide the means of suicide } \\
\text { by dispensing a prescription for use in PAS, as he/she should try to } \\
\text { preserve life }\end{array}$ & $57(32 \%)$ & $35(20 \%)$ & $87(48 \%)$ & 179 \\
\hline 9. & $\begin{array}{l}\text { It is appropriate for a physician not to inform a pharmacist about } \\
\text { the nature of a prescription intended to be used in PAS }\end{array}$ & $67(38 \%)$ & $34(19 \%)$ & $77(43 \%)$ & 178 \\
\hline 10. & $\begin{array}{l}\text { It is a pharmacist's right to know when his/her actions directly } \\
\text { contribute to the planned death of a patient }\end{array}$ & $95(53 \%)$ & $34(19 \%)$ & $49(28 \%)$ & 178 \\
\hline 11. & $\begin{array}{l}\text { It is appropriate for a pharmacist to refuse to dispense a } \\
\text { prescription if he/she knows that it is to be used in PAS }\end{array}$ & $97(54 \%)$ & $38(21 \%)$ & $44(25 \%)$ & 174 \\
\hline 12. & $\begin{array}{l}\text { There would be a danger that PAS could be abused to answer } \\
\text { social problems, by encouraging those who are seen as a burden on } \\
\text { society to take the "quick exit" }\end{array}$ & $108(60 \%)$ & $28(16 \%)$ & $43(24 \%)$ & 179 \\
\hline 13. & $\begin{array}{l}\text { PAS should remain an unofficial matter between physician and } \\
\text { patient, and as such, legislation is unnecessary }\end{array}$ & $58(33 \%)$ & $31(17 \%)$ & $88(50 \%)$ & 177 \\
\hline 14. & There needs to be a change in the law to allow PAS in Britain & $79(44 \%)$ & $41(23 \%)$ & $58(33 \%)$ & 178 \\
\hline 15. & $\begin{array}{l}\text { Legislating for PAS is necessary, as it means that it will be } \\
\text { regulated and cases dealt with appropriately }\end{array}$ & $91(52 \%)$ & $40(23 \%)$ & $45(25 \%)$ & 176 \\
\hline 16. & $\begin{array}{l}\text { The advent of PAS in this country would harm the public's } \\
\text { perception of pharmacists }\end{array}$ & $45(25 \%)$ & $47(26 \%)$ & $87(49 \%)$ & 179 \\
\hline
\end{tabular}

indicated that they did have some form of interest or involvement in ethics. Finally, participating pharmacists were asked to indicate whether or not their ethnic or religious background was a consideration in terms of how they dealt with ethical issues in practice. Almost three-quarters (130) $(72.6 \%)$ answered "no" to this question, five left the question blank and $44(24.6 \%)$ answered "yes". (Further statistical information is available from the authors.)

VIEWS OF PHARMACISTS

Tables 1 and 2 show respondents' views on the attitudinal statements. For clarity, the strongly agree and agree categories have been combined, as have the strongly disagree and disagree categories. In table $1,70 \%$ of respondents agreed with statement 1 that it was a patient's right to take his/her own life. Agreement reduced to $57 \%$ when asked about extending the right to choose to die to include the right to involve one's physician (statement 2). Agreement fell further to $45 \%$ when respondents were asked in statement 3 whether it was appropriate for a patient to use prescription medicines to commit suicide. As well as agreement declining with each successive question, the numbers disagreeing increased $(21 \%, 28 \%$ and $33 \%$ respectively) as did the numbers uncertain $(9 \%, 15 \%$ and $22 \%$ respectively). In statement $12,60 \%$ of respondents held the view that there would be a danger that PAS could be abused to answer social problems, by encouraging those who are seen as a burden on society to take the "quick exit". A minority of $33 \%$ felt that PAS should remain an unofficial matter between physician and patient, making legislation unnecessary (statement 13) with $44 \%$ agreeing that there needs to be a change in the law to allow PAS in Britain (statement 14).

Statements 5 to 11 in table 1 dealt with the professional rights and responsibilities of doctors and pharmacists. Whilst $46 \%$ of respondents reported that it was a pharmacist's professional responsibility to dispense a prescription for use in PAS (statement 7 ), only $29 \%$ of respondents held the view that it was a physician's professional responsibility to issue a prescription for use in PAS (statement 5). Thirty-eight per cent of respondents reported that they thought it appropriate for a physician not to inform a pharmacist about the purpose of a prescription to be used in PAS (statement 9). This is contrasted with the $53 \%$ of respondents who felt it was a pharmacist's right to know when his/her actions directly contributed to the planned death of a patient (statement 10). Finally, $54 \%$ of respondents stated that it was a pharmacist's right to refuse to dispense a prescription if he/she knew that it was to be used in PAS (statement 11).

The last series of statements were framed in the context of PAS having been legalised (table 2). 
Table 2 Views of pharmacists on PAS

\begin{tabular}{|c|c|c|c|c|c|}
\hline \multicolumn{2}{|c|}{ Statements } & \multirow{2}{*}{$\begin{array}{l}\text { Yes } \\
\qquad 161(90 \%)\end{array}$} & \multirow{2}{*}{$\begin{array}{l}\text { Don't Know } \\
8(5 \%)\end{array}$} & \multirow{2}{*}{$\begin{array}{l}\text { No } \\
9(5 \%)\end{array}$} & \multirow{2}{*}{$\begin{array}{l}\text { Total number } \\
178\end{array}$} \\
\hline 17. & $\begin{array}{l}\text { The RPSGB code of ethics should contain a protocol } \\
\text { advising pharmacists how to correctly comply with any } \\
\text { new law on PAS }\end{array}$ & & & & \\
\hline 18. & I would knowingly dispense a prescription for use in PAS & $88(49 \%)$ & $48(27 \%)$ & $43(24 \%)$ & 179 \\
\hline 19. & $\begin{array}{l}\text { As a pharmacist presented with a prescription for use in } \\
\text { PAS, I would want to know its intended use }\end{array}$ & $105(59 \%)$ & $29(16 \%)$ & $45(25 \%)$ & 179 \\
\hline 20. & $\begin{array}{l}\text { The conscience clause in the RPSGB code of ethics } \\
\text { should be extended to allow pharmacists who wish to } \\
\text { object to being involved in PAS to do so }\end{array}$ & $160(89 \%)$ & $10(6 \%)$ & $9(5 \%)$ & 179 \\
\hline
\end{tabular}

Forty-nine per cent of respondents said they would knowingly dispense a prescription for use in PAS (statement 18). A comparable $41 \%$ of respondents felt that, as a pharmacist presented with a prescription for use in PAS, they were either unsure $(16 \%)$ or would not want to know $(25 \%)$ its intended use (statement 19). Finally, 90\% of respondents felt that the professional code of ethics of the Royal Pharmaceutical Society of Great Britain should contain a protocol advising pharmacists how to comply correctly with any new law on PAS Further, $89 \%$ agreed that the conscience clause in the code of ethics should be extended to allow pharmacists who wish to object to being involved in PAS to do so. The questionnaire also provided space for additional comments. Just over one third (65 or $36 \%$ ) of respondents did so.

THE INFLUENCE OF RESPONDENTS' BACKGROUND A composite score was derived from the responses to the first eight statements. These statements were selected because they were felt to assess the respondents' agreement or disagreement with PAS Respondents were given a " 1 " for a strongly disagree up to a " 5 " for strongly agree. The range included the lowest possible minimum of eight and the highest possible maximum of 40 . The mean score was 24.7. The score obtained was used to compare levels of agreement with PAS with various demographic and background characteristics. The characteristics that were examined were mailing [first, second or third], gender, year of registration, practice setting and ethnic/religious background.

There was no difference between the level of agreement with PAS and the mailing from which the different responses were received, the year of registration as a pharmacist or the practice setting in which different respondents worked. However, there was a significant association between the level of agreement with PAS and the gender of the respondent $(p=0.028)$ and the declaration of an ethnic/religious background $(p=0.00005)$. This suggested that females were more favourable towards PAS than males and those who declared an ethnic/religious background of consideration in their dealings with ethics were significantly less likely to favour PAS.

\section{Discussion}

The questionnaire had a response rate, 56\%, typical of research in this area. ${ }^{11}$ This, and the relatively high level $(36 \%)$ of additional comments, suggests that the issues raised by the questionnaire were of interest to many British community pharmacists. Demographics were approximately matched for gender and year of registration. However, practice setting, ethnic/religious background or interest in ethics could not easily be estimated for the non-respondents and so comparison with nonrespondents was not possible. It is possible that they did not respond because they were particularly anti- or pro-PAS. There is, therefore, the possibility that the respondents were biased in some way.

The debate surrounding PAS continues to focus primarily on the rights and responsibilities of the patient and physician. However, PAS raises questions about the rights and responsibilities of the pharmacist in PAS irrespective of whether she or he is an informed and fully consenting participant or not. Comparable to physicians, are pharmacists compelled to follow the Hippocratic dictum primum non nocere (first do no harm) ${ }^{12}$ or the Donabedian model which advocates that the patient is the best judge of his or her own welfare $?^{13}$ Does a pharmacist have a legitimate right to deny a patient a pharmaceutical service because his or her conscience does not allow active cooperation in PAS? Should a pharmacist's personal beliefs impact on a patient requesting PAS from a health care professional?

On the issue of the patient's right to take his or her own life, $70 \%$ of respondents felt that the patient did have the right to choose to end his or her own life. This was similar to the American findings, ${ }^{3}$ suggesting a similar attitude between British and US pharmacists on this issue. Extending the right to die to the right to be assisted to die by a physician, fewer respondents $(57 \%)$ were in agreement, which fell to $30 \%$ agreeing that it was appropriate for prescription medicines to be used by a patient to commit suicide as in PAS. The level of agreement with a patient's right to choose to die decreased with the involvement of other people and the use of prescription medicines in the process, suggesting that respondents were less in favour of a patient's right to choose to die the more the scenario involved other people. It may suggest that respondents saw suicide as a conditional, rather than an absolute right. Whilst $57 \%$ of respondents thought it was a patient's right to choose to die with the assistance of his or her physician substantially fewer respondents $(29 \%)$ expressed the view that 
they believed it to be a physician's responsibility to take part in PAS by issuing a prescription. This makes a distinction between a patient's right and a professional responsibility. This may suggest that respondents saw a difference between a patient having a right to a particular health care service and necessitating every health care professional to provide that service.

\section{Significant association}

Unlike the two American PAS studies, this study found women $(n=64)$ to be significantly more likely to favour PAS than their male counterparts $(n=115) .{ }^{34}$ More in line with expectations, there was a significant association between those who declared a religious and/or ethnic background $(\mathrm{n}=44)$ and a less favourable attitude to PAS. It is not surprising that a religious background should exert an influence as most of the world's major religions are opposed to euthanasia and, by extension, PAS. The Rupp and Isenhower ${ }^{3}$ and the Vivian study $^{4}$ also found religious affiliation to be influential in the pharmacists' attitudes towards PAS. However, the age of the pharmacist was found to be a significant factor in both these studies, although not in the present study.

Whilst $46 \%$ of respondents considered it was a pharmacist's professional responsibility to dispense a prescription for use in PAS, substantially fewer respondents $(29 \%)$ felt that writing such a prescription constituted a professional responsibility for a physician. Does this difference suggest that pharmacists feel they have a different duty of care towards patients or more of a duty to do as they are told? It may be that many community pharmacists perceive their influence in health care as being very much secondary to that of the doctor and/or the patient. Thus they may see their involvement in the provision of medicines as indicated on a doctor's prescription as something of an inevitability in a process already in motion. This may be quite different from the perceived responsibility when a pharmacist initiates therapy by counter-prescribing a pharmacy-only medicine. Involvement in decisions about health care are likely to be more passive in the case of dispensing a prescription, than when counter-prescribing. Equally, the answers to this question could reflect a limitation of wording where other options, such as entering into a discussion with the prescriber or patient or seeking additiona information elsewhere, were not allowed for within the description.

Interestingly, 38\% felt it was appropriate for a physician not to inform a pharmacist about the nature of a prescription intended for use in PAS. In other words $38 \%$ believed it correct that the pharmacist should be kept in the dark where PAS was concerned. It is possible that the pharmacist considers patient confidentiality to be paramount. As a member of the multidisciplinary health care team the inclusion of the pharmacist in this process should only help to improve the outcome for the patient. It is also possible that the pharmacist is trying to escape responsibility with a "what you don't know can't hurt you" approach. The model of pharmaceutical care put forward by Hepler and Strand ${ }^{8}$ firmly places responsibility for defining and achieving patient outcomes in health care on the pharmacist's shoulders. In the current age of professional enlightenment, it is unacceptable for the pharmacist not to be involved in patient care. More damaging still is the finding that $25 \%$ of pharmacists would not wish to know the purpose of a prescription for use in PAS. This does not reflect well on a profession with aspirations to take on new professional roles and responsibilities and raises the question whether it is a profession-wide sentiment or particular to just community pharmacists. Future research might usefully determine if this view is similarly prevalent among hospital pharmacists. Hospital pharmacists may be more likely to have contact with terminally ill patients and, potentially, to be involved in more medicine management roles beyond dispensing.

The current guidance in the pharmaceutical profession's code of ethics (CE-RPSGB) allows for pharmacists to refuse to dispense certain items because of their personal convictions or religious beliefs. This conscience clause does not, at the present time, extend to drugs that could be used as part of PAS. The vast majority of pharmacists $(89 \%)$ supported an extension of this clause in the event of legalisation of PAS and $90 \%$ wanted a protocol in place. Both the American and Australian Medical Associations had policy statements on PAS before legislation on PAS was passed ${ }^{14}{ }^{15}$ as does the American Pharmaceutical Association. ${ }^{16}$ The medical and nursing professions in the UK similarly have issued policy statements on PAS and euthanasia. The pharmaceutical profession should be similarly prepared with a policy statement and a protocol for practice. This would ensure the provision of a pharmaceutical service to the patient while respecting the personal beliefs of the pharmacist.

\section{Conscientious objection}

Ultimately, for a pharmacist to cooperate in PAS by dispensing the means to commit suicide once legalised, would become a matter for each individual professional to decide. Any law or code of ethics requiring every pharmacist to dispense prescriptions for PAS would clearly be unjust, as it denies to the pharmacist, who is a professional not devoid of personal conscience, the right to make a conscientious objection. Such a courtesy is extended in many circumstances to nursing and medical colleagues. Pharmacists should be no exception. The decision a pharmacist comes to regarding his or her part in the process of care leading to the death of a patient in PAS should be an informed one. There is a need for bioethics to be taught as a core subject in the pharmacists' undergraduate degree course in UK schools of pharmacy, in the same way in which medical ethics is planned to be included in the core curriculum in UK medical schools. ${ }^{17}$ In order to allow all pharmacists to come to such an informed decision wide debate should be encouraged both within the profession of pharmacy 
as well as between patients' rights groups and the medical profession. Any discussions on PAS which do not involve input from the profession of pharmacy fail to recognise the part which pharmacists play in PAS and simultaneously fail to acknowledge the unique perspective which the pharmaceutical profession has on the supply and effective use of medicines. At the same time, the profession of pharmacy must recognise, and act on, its professional duty to encourage debate on the issue of PAS.

\section{Conclusion}

Pharmacists in this study view their professional responsibility towards PAS to be different from that of the physician. Pharmacists view their role as more obligatory, in having to provide the supply of drugs to be used in PAS, whereas the physician has more autonomy to refuse such a patient request. Pharmacists are currently advancing into new roles and responsibilities to provide optimum patient care. They wish to be regarded as key members of primary health care teams. Yet a proportion of the respondents in this study have no desire to take responsibility for their actions and prefer to remain in ignorance of the true purpose of a prescription for PAS. The findings suggest that debate should be encouraged within the profession on the subject of PAS. The RPSGB should consider developing a practice protocol for PAS as well as extending the conscience clause of the code of ethics of the Royal Pharmaceutical Society of Great Britain in the event of new legislation. Such measures would allow the efficient provision of the pharmaceutical service whilst at the same time respecting the personal beliefs of those who object to cooperating in the taking of a human life.

\section{Appendix I}

The Questionnaire:

To illustrate physician-assisted suicide (PAS), consider the following example:

Janet Smith is a 50 -year-old school-teacher. She is the mother of two teenage children. She has terminal cancer and is not expected to live for more than 12 months. After discussing the matter with her husband and family, she decides to ask the family GP to help her to die. Her GP agrees to prescribe enough barbiturates and a muscle relaxant for her so that she can die at a time and place decided by her. Janet goes to her local community pharmacy and obtains the prescription medicines from the pharmacist. She takes the prescribed barbiturates and the muscle relaxant and dies in the expected way.

This is an example of physician-assisted suicide. Janet's GP has assisted her to end her own life by means of a prescription for a lethal dose of drugs dispensed by a community pharmacist. The physician does NOT administer the lethal dose in physician-assisted suicide as would happen in euthanasia.
Rate your agreement with the following statements (strongly agree to strongly disagree on a five-point scale). Please assume that the law is still in its present form as regards to PAS:

1. A patient has the right to choose to end his/her own life.

2. A patient has the right to choose to die with the assistance of his/her physician.

3. It is appropriate for a patient to use prescription medicines to commit suicide.

4. A prescription medicine should not be used to end a life prematurely.

5. A physician has a professional responsibility to alleviate suffering by issuing a prescription for physician-assisted suicide (PAS).

6. It is a physician's professional responsibility not to act in such a way that a life is ended prematurely as in PAS.

7. A pharmacist has a professional responsibility to dispense a prescription to be used in PAS, if that is what the patient wants.

8. It is inappropriate for a pharmacist to provide the means of suicide by dispensing a prescription for use in PAS, as he/she should try to preserve life.

9. It is appropriate for a physician not to inform a pharmacist about the nature of a prescription intended to be used in PAS.

10. It is a pharmacist's right to know when his/her actions directly contribute to the planned death of a patient.

11. It is appropriate for a pharmacist to refuse to dispense a prescription if he/she knows that it is to be used in PAS.

12. There would be a danger that PAS could be abused to answer social problems, by encouraging those who are seen as a burden on society to take the "quick exit".

13. PAS should remain an unofficial matter between physician and patient, and as such, legislation is unnecessary.

14. There needs to be a change in the law to allow PAS in Britain.

15. Legislating for PAS is necessary, as it means that it will be regulated and cases dealt with appropriately.

16. The advent of PAS in this country would harm the public's perception of pharmacists.

Please assume that the law has now changed and that PAS is now legal: (Yes, Don't know, No).

17. The RPSGB code of ethics should contain a protocol advising pharmacists how to correctly comply with any new law on PAS.

18. I would knowingly dispense a prescription for use in PAS.

19. As a pharmacist presented with a prescription for use in PAS, I would want to know its intended use. 
20. The conscience clause in the RPSGB code of ethics should be extended to allow pharmacists who wish to object to being involved in PAS to do so.

$\operatorname{Sex}(\mathrm{M} / \mathrm{F})$

Year registration

Current practice setting (independent, multiple (less than 30 shops), multiple (more than 30 shops)

Do you have any interest or involvement in ethics as a subject, for example, ethics committees or teaching?

Is your ethnic/religious background a consideration in terms of how you deal with ethical issues in practice? (If yes, how)

Additional comments

Timothy R G Hanlon, MSc, MRPharmS, is a Hospital Pharmacist and a Former MSc student. Marjorie $C$ Weiss, MSc (Clinical Pharmacy), MSc (Social Research Methods), DPhil, MRPharmS, is Lecturer in Primary Care, University of Bristol, Whiteladies Road, Bristol BS8 2PR. Fudith Rees, PhD, MRPharmS, is Senior Lecturer in Pharmacy Practice, School of Pharmacy and Pharmaceutical Sciences, University of Manchester, Manchester. Address for correspondence: $M$ $C$ Weiss.

\section{References}

1 e-Hansard from The Houses of Parliament web site@ http://www.parliament.the-stationery-office.co.uk/pa/cm/

cmhansard.html.

2 Euthanasia in Holland from the Dutch Voluntary Euthanasia Society web site @ http://www.netlink.co.uk/users/vess/ Society we
3 Rupp MT, Isenhower HL. Pharmacists' attitudes toward physician-assisted suicide. American fournal of Hospital Pharmacy 1994;51:69-74.

4 Vivian JC, Slaughter RL, Calissi P. Michigan pharmacists' attitudes about medically assisted suicide. Fournal of Michigan Pharmacy 1993;31:490-5.

5 Royal Pharmaceutical Society of Great Britain. Medicines, ethics and practice - a guide for pharmacists. London: RPSGB, 1998.

6 Pharmacy: A report to the Nuffield Foundation. London: Pharmacy: A report to the
Nuffield Foundation, 1986.

7 Leach RH, Wakeman A. An evaluation of the effectiveness of community pharmacists working with GPs to increase the cost-effectiveness of prescribing. Pharmaceutical fournal 1999; 263:206-9.

8 Hepler CD, Strand LM. Opportunities and responsibilities in pharmaceutical care. American fournal of Hospital Pharmacy 1990;47:533-42.

9 Brushwood DB. The pharmacist's expanding legal responsibility for patient care. Fournal of Social and Administrative ity for patient care. four

10 Appelbe GE, Wingfield J. Pharmacy law and ethics [6th ed]. London: The Pharmaceutical Press, 1997: 240.

11 Weiss MC, Cantrill JA, Nguyen HL. Pharmacists' and pre-registration graduates' views of proxy consultations. International fournal of Pharmacy Practice 1998;6:121-6.

12 Uhlmann M. Western thought on suicide: from Plato to Kant. In: Uhlmann M, ed. Last rights? Assisted suicide and euthanasia debated [1st ed]. Michigan, USA: Eerdmans Publishing Company, 1998

13 Rupp MT. Physician-assisted suicide and the issues it raises for pharmacists. American fournal of Health-Systems Pharmacy pharmacists. Amer

14 American Medical Association. E-2.211 Physician-assisted suicide. American Medical Association Ethical Opinion, 1994 June.

15 Australian Association for Hospice and Palliative Care. Care of severely and terminally ill patients. Australian Medical Association position statement, 1996 (amended November 1997).

16 American Pharmaceutical Association. 1997-8 Policy Committee Report. Pharmacist conscience clause. 1998 March.

17 Consensus statement by teachers of medical ethics and law at UK medical schools. Teaching medical ethics and law within medical education: a model for the UK core curriculum. Yournal of Medical Ethics 1998;24:188-92. 